\title{
GESTIÓN DE LA INNOVACIÓN POR MICROEMPRESARIOS DEL CENTRO DE DESARROLLO DE NEGOCIOS DE TALCA, CHILE
}

\author{
MANAGEMENT OF INNOVATION BY MICROENTERPRISES OF \\ THE BUSINESS DEVELOPMENT CENTER OF TALCA, CHILE
}

\author{
Francisca Gajardo Leyton, ' Miguel A. Bustamante U. 2,*
}

1 Magister en Gestión Tecnológica, Universidad de Talca, Dos Norte 685, 3465548 Talca, Chile.

2 Facultad de Economía y Negocios, Universidad de Talca, Dos Norte 685, 3465548 Talca, Chile.

E-mail: gajardo.fran@gmail.com; mabu@utalca.cl

(*) Autor de Correspondencia

RESUMEN

ABSTRACT

El presente trabajo determina los factores cualitativos que definen la gestión de la innovación por parte de los microempresarios del centro de desarrollo de negocios de Talca, Chile. Para ello se analizó la percepción de la innovación y las acciones que se despliegan para su desarrollo. Se condujo un estudio con enfoque y métodos cualitativos, aplicando como instrumento de recolección de datos una entrevista semi-estructurada a microempresarios dueños de sus respectivos emprendimientos que se han asesorado en el centro de desarrollo de negocios de Talca, Chile por un periodo de al menos, un año. Los resultados del estudio permiten afirmar que la innovación es considerada por los entrevistados como importante y necesaria, y están dispuestos a innovar pese a los riesgos que esta práctica pueda involucrar a nivel tanto empresarial como personal. Además, se verifica que los entrevistados se mueven en el mercado de manera independiente, incluso solitaria, utilizando internet como principal fuente de información para innovar. Complementariamente, se ratifica el hecho que el factor recurso financiero está presente de forma transversal en todas las dimensiones y categorías analizadas y además, no cuentan con la información necesaria, ni con el apoyo o la guía suficiente para innovar en sus empresas. Finalmente, es de especial interés abordar el impacto de las políticas públicas de fomento a la innovación, especialmente dirigida al segmento de los microempresarios.

Palabras clave: innovación; microempresas; estrategias; percepciones; obstáculos; facilitadores.
This paper determines the qualitative factors that define the management of innovation by microentrepreneurs of the business development center of Talca, Chile. For this, the perception of innovation and the actions that are deployed for its development were analyzed. A study with qualitative approach and methods was conducted, applying as a data collection instrument a semi-structured interview to microentrepreneurs who owned their respective ventures who have been advised in the business development center of Talca, Chile for a period of at least, one year. The results of the study allow us to affirm that innovation is considered by the interviewees as important and necessary, and they are willing to innovate despite the risks that this practice may involve at both business and personal level. In addition, it is verified that the interviewees move in the market independently, even alone, using the Internet as the main source of information to innovate. Complementarily, the fact that the financial resource factor is present in a transversal way in all the dimensions and categories analyzed is ratified and, in addition, they do not have the necessary information, nor the support or sufficient guidance to innovate in their companies. Finally, it is of special interest to address the impact of public policies for promoting innovation, especially aimed at the microentrepreneurs segment.

Keywords: innovation; microenterprises; strategies; perceptions; obstacles, facilitators. 


\section{Introducción}

Desde los años noventa de manera sistemática se han hecho grandes esfuerzos en Chile por fomentar el desarrollo de nuevas empresas (CEPAL Naciones Unidad, 2002) y consecuentemente asumiendo el signo de los tiempos actuales, en los últimos años se observa que el foco desde las instituciones públicas ha sido el fomento de la innovación en las organizaciones empresariales en todos sus diversos tamaños (Dini \& Stumpo, 2018). Es así como, desde la perspectiva de la innovación, se aprecia que en las industrias, la innovación es el camino recomendado para estimular el desarrollo de los países, por lo que se ha hecho necesario potenciarla esta perspectiva tanto, desde la formación inicial educativa y profesional de las personas como desde la mirada de las empresas, independientemente de su tamaño, con especial focalización en las empresas de menor tamaño, EMT (Ffrench-Davis \& Díaz, 2019).

Dentro del grupo de las EMT están las microempresas, las cuales representan un $44,4 \%$ de las empresas establecidas en el país (Ministerio de Economía, Fomento y Turismo, 2019) teniendo una alta presencia en la economía y por lo tanto, una activa presencia en términos de productividad y generación de empleo (Paula, Alvarez, \& Rezende, 2018), en consecuencia, es en base a esto que surge el interés de enfocar el estudio de la innovación en este segmento en particular de las organizaciones empresariales, en razón de las cuales se plantean las siguientes preguntas de investigación, a decir, ¿Cómo definen la innovación los sujetos del estudio?, ¿Qué tipo de acciones y estrategias desarrollan los sujetos de estudio para innovar en sus actividades productivas? y ¿Cuáles son los principales obstáculos y/o facilitadores que enfrentan los microempresarios cuando intentan innovar?

\section{La Innovación}

De acuerdo con el Manual de Oslo de la OCDE en su versión del año 2005, una empresa innovadora se define como aquella que ha logrado introducir "algo nuevo" o significativamente mejorado para el contexto particular de esa empresa. Ese "algo" puede ser un producto, servicio, método de comercialización, organizativo, las prácticas internas de la empresa, o su relaciones externas (OCDE, 2005). Complementariamente, el Consejo Nacional de Innovación para la Competitividad en Chile (Consejo Nacional para la Competitividad, 2010) indica que la innovación es un fenómeno complejo y de carácter sistémico. Esto quiere decir que en él influyen diversos factores y actores que interactúan con el objeto de facilitar este proceso del cual se nombra como protagonista a las empresas, que son las que finalmente llevarán la innovación al mercado. Además, el Estado está fuertemente comprometido con el fomento de la innovación a través de cuatro ejes estratégicos que buscan hacerse cargo de generar un marco que propicie incentivos para que las empresas innoven (Ministerio de Economía, Fomento y Turismo, 2018).

\section{El empresario innovador}

Para Schumpeter (1942), una persona se define como empresario innovador cuando es capaz de tomar la iniciativa y realizar cambios y mezclas en los medios de producción. Debe ser pionero en crear nuevos productos, procesos o nuevas formas de organización, sin preocuparse del riesgo. Para asumir el riesgo están los directores, ejecutivos o accionistas, a los que diferencia de los empresarios llamándolos "hombres de negocios" pues estos no son quienes se encargan de generar las nuevas ideas (Montoya, 2004). En este sentido, el empresario innovador es capaz de detectar oportunidades de negocio aún en ambientes que puedan ser percibidos como entornos o situaciones de aparente caos, contradicción, dificultades o inclusive amenazas (Cancino, Bonilla, \& Vergara, 2015), ya que está permanentemente informado sobre las novedades y cambios en su sector.

Así mismo, las empresas predominantes en Chile son las pequeñas y medianas, representando un $51,9 \%$ del total de empresas. Le siguen las microempresas con un $44,9 \%$ mientras que las grandes solamente representan un 3,2\% (Ministerio de Economía Fomento y Turismo, 2017). Además, por su alta presencia en el total de las empresas en Chile, las microempresas junto con las Pymes juegan un papel importante en la economía del país, generando nuevos empleos y activando la competencia en el mercado. Sin embargo no existen cifras oficiales relacionadas a la innovación en las microempresas ya que no están incluidas en la Encuesta Nacional de Innovación que aplica el Ministerio de Economía junto al Instituto Nacional de Estadísticas bienalmente (Instituto Nacional de Estadísticas, 2019). Por otro lado, los Centros de Desarrollo de Negocios son una iniciativa impulsada por el Servicio de Cooperación Técnica, SERCOTEC que busca potenciar las EMT a través de servicios de asesoría personalizada y capacitación en diversas áreas de la gestión de las empresas. Otros servicios incluyen vinculaciones con entidades que les provean de nuevos conocimientos, herramientas, financiamiento y la articulación con el ecosistema productivo local (Centros Chile Sercotec, 2018)

Sintetizando, se trata de asumir cuatro ejes de acción que buscan, a) promover una cultura de innovación que llegue a empresas de todos los tamaños, b) potenciar la productividad de nuevas áreas que permitan diversificar la economía, c) fortalecer la colaboración entre universidad y empresa a través de la transferencia tecnológica y d) potenciar el impacto de las políticas públicas para el fomento de la innovación y asegurar el seguimiento y evaluación de los recursos que se destinan a las diversas iniciativas.

Finalmente, el presente estudio pretende dar respuesta a las preguntas de investigación con especial énfasis en una visión holística que busca comprender de manera profunda la realidad de microempresarios dueños y gestores se sus respectivos emprendimientos, cuyas entidades tienen domicilio comercial en la provincia de Talca, Chile y que son usuarias del Centro de Desarrollo de Negocios en el cual han recibido servicios de asesoría especializada por a lo menos un año, obteniendo de esta forma información de primera fuente.

\section{Metodología}

Este estudio se abordó desde la perspectiva y métodos cualitativos con el propósito de lograr un acercamiento a las visiones y experiencias de un grupo de sujetos, lo que plantea un fenómeno de la vida real sobre el cual la investigadora tiene escaso control (Chetty, 1996; Yin, 2009) que no sería posible lograr con procedimientos cuantitativos dado la 
naturaleza de los objetivos planteados (Cornejo, Carmona, Besoaín, \& Manosalva, 2012), se realizó en consecuencia un estudio descriptivo orientado a generar una cierta hipótesis explicativa de la realidad como plantean Rothman y Greenland (1998). En este sentido se realizó un estudio de casos múltiple (Yin, 2009) a partir de emprendedores que no pretende ser una muestra representativa, como es el caso de los estudios cualitativos estadísticos, si no más bien una muestra teórica (Eisenhardt, 1989) que tiene como fin que los resultados puedan ser transferibles a otras situaciones o realidades similares (Maxwell, 1998; Rialp, 1998).

\section{Recolección de datos cualitativos}

Los participantes del estudio fueron seleccionados a partir de un muestreo intencionado no probabilístico (Glaser \& Strauss1967) dado que el perfil de los sujetos que serían parte del estudio fue determinado previamente según parámetros vitales para el cumplimiento del objetivo de la investigación (Patton, 1990). Los casos de contacto, fueron como un proyecto de investigación exploratorio que examina e indaga el tema objetivo (Maxwell 1998), como es la innovación en las microempresas, específicamente de aquellas cuyo objetivo es generar nuevo conocimiento que permita el surgimiento de nuevas preguntas de investigación que pueda dar origen a nuevas líneas investigativas que sean abordadas desde perspectivas analíticas complementarias.

El universo de sujetos susceptibles a ser parte del estudio debió cumplir con las siguientes características, hombres o mujeres de 25 años o más, dueños de microempresas de diversos rubros, formalizadas, con domicilio tributario en la ciudad de Talca; ser clientes activos del Centro de Desarrollo de Negocios con antigüedad de más de un año; que hayan desarrollado durante el último año cualquier tipo de innovación en sus negocios; que las empresas cumplan con el perfil de haber nacido a partir de una idea $u$ oportunidad de negocio; en esta línea, se excluyen aquellas empresas nacidas de emprendimientos por necesidad, asumiendo en consecuencia, que es mediante las primeras empresas, derivadas de oportunidades que es posible hacer cambios estructurales en la economía (Gries \& Naude, 2008; Lewis, 1954; Ranis \& Fei, 1961). Finalmente se escogieron como casos de estudio seis hombres y seis mujeres dueños y dueñas de micro y pequeñas empresas que accedieron a ser parte del estudio, cuyas edades fluctúan entre los 29 a 60 años. El número de entrevistados se definió por el punto de saturación (Eisenhardt, 1989), es decir cuando un número mayor de entrevistados no otorga información nueva relevante a los objetivos de investigación (Martínez-Salgado, 2012).

\section{Instrumento de recogida de datos}

El instrumento utilizado para obtener los datos fue la entrevista en profundidad semi-estructurada dado su grado de flexibilidad suficiente para poder adaptarse a los sujetos en el caso de ser necesario aclarar conceptos, despejar opiniones imprecisas haciendo contra preguntas que surgen durante la entrevista de manera espontánea, es decir, el método cualitativo aquí descrito permite "salir del guion" con el propósito de motivar a los entrevistados a expresar sus ideas de la forma más completa posible para así asegurar la obtención de información clave para el estudio (Díaz-Bravo, Torruco-García, MartínezHernández, \& Varela-Ruiz, 2013). Conforme a esto, se realizaron preguntas abiertas con el objetivo de recabar datos consistentes tanto en lenguaje verbal y no verbal como también lenguaje visual, que son analizados mas tarde concentrándose en las vivencias de los sujetos de estudio, teniendo en cuenta que la investigadora es por sí misma un instrumento de recolección de datos cualitativos además de los recursos de apoyo que utiliza: cuaderno de campo, notas de campo y grabadora Shaw (1999) debido a la naturaleza subjetiva del análisis cualitativo. Esta postura permite luego realizar el análisis y vinculaciones de tendencias de las experiencias de los sujetos de estudio tal como ellos describen, experimentan y manifiestan su realidad relacionada con el tema de investigación (Sherman \& Webb, 1988).

Las entrevistas se llevaron a cabo en una sola sesión presencial en todos los casos con una duración de entre $45 \mathrm{y} 60$ minutos aproximadamente y fueron aplicadas entre la tercera semana de junio a la segunda semana de agosto del año 2018. Cada una de las entrevistas fue grabada en formato de audio en una aplicación de Smartphone con el consentimiento previo de los participantes del estudio.

\section{Proceso de análisis de los datos}

Para el análisis de datos primero se transfirió el material de audio a texto escrito con cuidado de que la transcripción sea lo mejor detallada e íntegra posible para cuidar los aspectos naturales de la expresión de los participantes, sus modismos, acentos, pausas, silencios, etc. Todos estos criterios permiten resguardar la recogida de, palabras, fonemas, dichos y expresiones particulares que aportan información para finalmente lograr la elaboración de un texto que totaliza y a través del cual se analiza e interpreta mediante la técnica de Análisis de Contenido definida por Krippendorff (1990) como aquella que, a partir de los datos recolectados, busca formular inferencias reproducibles y válidas para el contexto en que se desarrolla el proceso de investigación cualitativa que, según Uwe Flick (2004), es posible ser representado como un flujo constante desde la teoría conceptual a lo textual de forma recíproca.

El segundo paso para llevar a cabo el análisis fue leer varias veces las transcripciones de las entrevistas junto a las nota de campo relacionadas a cada una de estas, para luego definir a partir de la lectura crítica cuales serían los segmentos que tuvieran el contenido idóneo a los objetivos de la investigación para ser analizados en profundidad en la siguiente etapa. Los segmentos seleccionados, que en este caso son frases, conjunto de frases, oraciones o expresiones que se denominan completas a partir de un inicio a un punto seguido, punto a parte o pausa (finalización de la frase o expresión), son agrupados por temas o ideas similares. Así mismo, los segmentos se codificaron según las ideas que estos representan a criterio de la investigadora sin perder la perspectiva de los objetivos de la investigación y el marco teórico conceptual. De este modo, al codificar todos los segmentos se agruparon en las categorías a las que estaban relacionados. Complementariamente, la codificación permite que otros investigadores puedan validar los resultados de la investigación al poner a prueba el sistema de códigos, debiendo ser capaces de llegar a resultados similares siguiendo el mismo método (Strauss \& Corbin, 1990). 
En primer lugar, se busca interpretar las percepciones que tienen los emprendedores entrevistados con respecto a la innovación, desglosando desde este punto cómo es que definen y entienden el concepto de innovación, apreciaciones relacionadas al riesgo al momento de innovar y las motivaciones que los llevan a ello.

En segundo lugar, se hace una descripción sobre la indagación de las estrategias que los sujetos participantes dicen llevar a cabo cuando deciden innovar, teniendo en cuenta las acciones involucradas, como trabajan las vinculaciones con otras empresas, personas o instituciones públicas y cuales son sus principales fuentes para obtener apoyo o información.

Finalmente, se describen los principales factores del entorno que afectan la gestión de la innovación en este grupo de estudio, resultando tanto en obstáculos por una parte, como otros que indican han facilitado el cumplimiento de sus objetivos de innovar en sus empresas.

\section{Resultados}

Para conocer cual es el concepto que los entrevistados manejan con respecto a la innovación, se les hizo la pregunta: “¿Qué entiende usted por innovación?” produciendo respuestas inmediatas en todo los sujetos y aunque similares, variaron naturalmente en la extensión y complejidad de los términos utilizados. Al respecto, 8 de los entrevistados $(67 \%)$ relacionaron la innovación con el concepto de "algo nuevo" en la empresa. De estos, cinco (42\%) se refieren a lo nuevo como una creación que puede ocurrir dentro de la empresa, en la creación de un producto o servicio nuevo, o la creación de nuevos procesos. Estas definiciones se pueden asociar a la innovación del tipo disruptiva. Por otra parte, los otros tres entrevistados $(25 \%)$ que usan la palabra "nuevo" sugieren que la innovación involucra incorporar estos elementos nuevos externos a la empresa, lo que describen como la adquisición de tecnología o nuevos conocimientos.

Otro de los elementos presentes en la mayoría de las respuestas es que la innovación significa alejarse de las prácticas tradicionales con el objetivo de lograr la eficiencia y la optimización de los recursos. No es raro que para este grupo tenga un alto interés en la optimización de los recursos puesto que este segmento empresarial tiene como una de sus características la vulnerabilidad financiera. En torno a ello, tres empresarios (25\%) entrevistados manifestaron entender la innovación como un agente diferenciador que les permite atraer nuevos clientes y mejorar la competitividad con respecto a la competencia. Además, cinco de los entrevistados (42\%) incluyeron en sus definiciones el objetivo que tiene la innovación en las empresas, para lo que usaron conceptos como, agregar valor, optimizar un producto, servicio o proceso interno de la empresa.

Una expresión referencial de lo analizado se refiere en el párrafo siguiente:

"Poder optimizar o crear nuevos procesos. Lo tomo más como un proceso que un producto. Poder optimizar, crear un proceso nuevo para obtener un resultado mejor que lo que había hasta el momento."

G.B.35 años, hombre.
En el segmento de empresarios de mayor edad, 56 a 60 años, resultó más común encontrar en sus respuestas descripciones menos complejas. Al respecto, según estas respuestas, se puede inferir que es un concepto que no han terminado de comprender y del que se sienten lejanos. Incluso una de las respuestas incluye la expresión "una persona como yo" aludiendo a su edad, dando a entender que es la causa de que la innovación sea un tema de difícil comprensión. Además, si contrastamos las respuestas de los empresarios con la definición que postula el Manual de Oslo se puede apreciar que en general el entendimiento de este grupo de estudio se acerca bastante a esta definición y otras ampliamente aceptadas de innovación:

"Una innovación es la introducción de un nuevo, o significa tivamente mejorado, producto (bien o servicio), de un proceso, de un nuevo método de comercialización o de un nuevo método organizativo, en las prácticas internas de la empresa, la organización del lugar de trabajo o las relaciones exteriores"

(OCDE, 2005, pág. 56).

\section{En torno a la percepción de los riesgos}

Para comprender en profundidad las apreciaciones sobre innovación que tienen los sujetos de estudio, también se buscó indagar en lo que estos empresarios perciben como riesgos, es decir, cuales son sus mayores temores empresariales y personales al momento de innovar, partiendo de la premisa de que todo y cualquier innovación supone siempre un riesgo.

Frente a la pregunta "¿Qué cree usted que pone en riesgo al innovar?" hubo entrevistados que negaron que haya riesgo al momento de innovar en sus empresas. Sin embargo, incurrían en una contradicción inmediatamente al plantear que el riesgo era absolutamente necesario para llevar a cabo esa innovación, mencionando, entre otros riesgos importantes para los sujetos, aquellos que están presente en la mayoría de los casos, como es el hecho de perder la inversión realizada, ya que el capital que manejan las microempresas normalmente es acotado y el fracaso de la innovación podría poner en jaque la continuidad de la empresa. Otro riesgo mencionado transversalmente es el de perder clientes que ya tenían una proporción de ellos fidelizados, sin embargo, podrían perderse a causa de que algún cambio en los productos o la manera de prestar los servicios no sea bien recibido por su público objetivo, lo que se traduciría inmediatamente en una baja en las ventas y afectaría por consecuencia la estabilidad económica del negocio.

\section{Fuentes de acceso a la innovación}

La fuente principal de información declarada por los sujetos entrevistados es Internet. Esta es la "Red" dondelosmicroempresariosbuscanideas, tendencias, regulaciones. A veces incluso se comunican con otras personas en foros o similares para conocer lo que estos están haciendo independientemente que sean personas o empresas, e incluso, pudiendo ser del mismo rubro en Chile y el resto del mundo. A modo de ejemplo, una expresión en este sentido indica:

"Participo en foros y cosas donde salen ideas y la gente habla de tendencias, pero en general como en internet. Siempre estoy como, no solamente leyendo, si no que participando en foros o comento artículos de cosas de comunicación y marketing." 
Además de internet, los entrevistados reciben y valoran la información que puedan entregarle sus respectivos proveedores, a quienes, los reconocen como expertos en los productos que les ofrecen y confían en ellos para introducir nuevas herramientas tecnológicas en sus empresas. Seguidamente, otra fuente de información importante que indican algunos de los entrevistados es la necesidad que reconocen de dedicar espacio a la observación de la competencia de manera indirecta o presencial. De esta forma obtienen ideas o buscan detectar fallas en los productos o servicios que entregan sus competidores con el objetivo de mejorarlos.

\section{Estrategias de innovación}

Las estrategias que despliegan los microempresarios entrevistados cuando emprenden un proyecto de innovación de cualquier tipo que sea en sus respectivos negocios, son en general, desde la cautela tomando todos los resguardos posibles y necesarios para asegurar la continuidad de la empresa en caso de que la innovación falle. Lo primero que hacen es buscar ideas. Estas pueden provenir de diferentes fuentes que ya han sido descritas en este documento, pero principalmente son Internet, las nuevas exigencia de sus clientes y la observación de lo que hace la competencia. Aunque un par de personas aluden a que las ideas surgen de ellos mismos. La expresión representativa se manifiesta de la siguiente forma.

"Lo que pasa es que generalmente yo siento que el tema de la innovación es como que la misma demanda de los clientes te va obligando a hacerla. Porque son ellos en el fondo los que van exigiendo cosas. Esto te obliga a ti a investigar 'acerca de' y poder mejorarlo para poder of recer el servicio."

\section{L.A. 29 años, mujer.}

Es así como, una vez que tienen una idea para que sus empresas resulten innovadoras, se detienen a realizar un "análisis de factibilidad" que tiene por objetivo saber si la apuesta será rentable y beneficiosa para la empresa en el caso de ser exitosa. Los métodos utilizados para este análisis no siguen ningún método sistemático, si no que se refiere a una reflexión personal que hacen los dueños de las microempresas teniendo en cuenta sus conocimientos acerca de la innovación que quieren implementar, y el conocimiento empírico de a gestión de sus negocios.

El tercer paso necesario e ineludible es, generalmente, la necesidad de asegurar que financieramente sea posible llevar a cabo la innovación. Esto incluye contar con el respaldo necesario en el caso de que esta falle. Es por ello que, cuando ya se ha decidido implementar la innovación, en el caso de que esta sea un nuevo producto o servicio, los microempresarios hacen pruebas de mercado que les permitan tener feedback de los clientes o simplemente hacen una integración gradual de los productos o servicios, manteniendo los tradicionales de manera principal de manera de analizar el impacto de estos en el mercado objetivo. Además el producto tradicional sirve de gancho de lo que ya "esta probado" y tiene clientes fidelizados que los empresarios esperan que se atrevan a probar algo nuevo por la confianza que ya han ganado de ellos.

Finalmente, lo que se plantea en este punto es una interpretación del conjunto de respuestas entregadas por los entrevistados, por lo que el orden de los pasos no es necesariamente el que siguen todos los microempresarios, de hecho algunos no incluyeron en sus relatos todos estas etapas de manera explícita, pero en función del análisis se ha preferido inferir un orden a modo demostrativo.

\section{Vinculaciones para la innovación}

En términos generales, se evidencia de los diversos relatos una necesidad de generar vínculos y redes orientadas a la innovación. Al parecer, es un hecho que todos los entrevistados reciben servicios del Centro de Desarrollo de Negocios, que tiene como particularidad generar vínculos con otras instituciones, profesionales y empresas. Sin embargo es muy común entre este grupo de microempresarios que no busquen relacionarse con otras empresas del rubro o similares.

Algunos empresarios se relacionan con otras empresas utilizando estrategias pasivas indirectas, por ejemplo usando foros en Internet o participando de capacitaciones donde puedan observar y escuchar experiencias de otros emprendedores. El valor que perciben en este tipo de relación con otras empresas es porque tienen acceso a la información la manera en que otros están desarrollando actividades productivas similares a ellos y como están solucionando los problemas que son similares a los suyos.

"El Centro (de Desarrollo de Negocios) me ha ayudado en temas de capacitación, y bueno navego harto por internet, busco ideas. También hay una página en Gmail que se llama OTEC de Chile que están... debe haber más de dos mil OTEC y ahí si uno saca ideas."

J.T. 56 años, mujer.

Motivación para innovar

Las motivaciones que los sujetos entrevistados revelan cuando responden a la pregunta: “¿Qué le motiva a innovar?" tienen como denominador común el aumento de la rentabilidad y competitividad de la empresa, que es un deseo inherente a cualquier empresario y no sería nada nuevo de descubrir a través de un estudio cualitativo. Esta percepción, también se puede traducir de las motivaciones que plantean los empresarios como resultado de la presión que existe en los mercados en general de que "estancarse es morir", sobre todo aquellas empresas relacionadas a tendencias como es el caso de las agencias de publicidad. Al respecto, la apreciación de uno de los entrevistados dice:

"Es que refleja en realidad lo que una empresa de publicidad también busca, y a parte por un tema de renta bilidad. Si no hay innovación, hay menor capacidad o hay menor condición de poder lograr metas. O sea, si yo me quedo en el pasado no voy de la mano con lo que hoy esta en proceso para todo el mundo y voy a perder la oportunidad de hacer rentable mi empresa."

P.A.34 años, hombre.

Otros factores que revelan los relatos como motivos para afrontar los riesgos que implica innovar en las microempresas están asociados a impulsos y convicciones personales que se pueden explicar por las características típicas de los empresarios innovadores y también por un argumento "Darwiniano" que plantea la necesidad intrínseca del ser humano de crear, mejorar, avanzar y adaptarse a los cambios del ambiente para sobrevivir. En tono a este punto uno de los entrevistados plantea: 
"Porque hace la diferencia también. Y por una cuestión de que no se po', de que sale de uno, digamos. ¿Cierto? Porque la competencia es súper importante, si no te mueres, pero no todo es competir.

J.T. 56 años, mujer.

\section{Obstáculos a la innovación}

El principal obstáculo que mencionan 11 de los 12 sujetos entrevistados (92\%) tiene relación con la apreciación del alto costo que tiene el hecho de innovar, y el poco acceso a recursos económicos que les permitan invertir en innovación, ya sea por generación de recursos por parte de la misma empresa, o de financiamiento de instituciones públicas, o inversionistas que pudieran interesarse en sus proyectos. Muchos de estos mismos empresarios relacionan la dificultad de financiar sus proyectos de innovación con la falta de guía o limitaciones para acceder a información relacionada al desarrollo de proyectos de innovación; no saben dónde acudir. Entre los relatos se destaca el siguiente:

"Yo creo que lo mismo el tema de sentirse sola. Yo creo que eso ha sido mi... porque de repente he querido hacer algo, pero ¿A quien acudo?, ¿Quién me puede ayudar? No hay alguien que esté como al 100 conmigo y diciendo "ya, yo te ayudo, podríamos hacer esto', no."

$$
\text { P.B. } 33 \text { años, mujer. }
$$

En menor medida se menciona también como un obstáculo la dificultad de dedicar tiempo a desarrollar la innovación en sus empresas, pero al mismo tiempo esto se relaciona nuevamente a la falta de recursos que deriva en incapacidad de contratar personal especializado o de apoyo. También se manifestó la dificultad de estos microempresarios para trabajar de manera constructiva con otras empresas de la competencia. Los perciben como un obstáculo para el desarrollo de sus negocios, en este caso por desacuerdos sobre las prácticas comerciales de los competidores que los afectarían.

\section{Facilitadores a la innovación}

La Mayoría de los entrevistados nombró explícitamente al Centro de Desarrollo de Negocios como un factor facilitador fundamental, tanto en el apoyo a la gestión en general del negocio, como en orientaciones para innovar. Especialmente los entrevistados valoran al Centro por la capacidad de generar nexos entre los empresarios con otras instituciones o empresarios, lo que les ha permitido generar redes de apoyo para incorporar nuevas herramientas en las empresas y/o sacar adelante sus proyectos de innovación. La forma en que lo expresan es la siguiente:

"Centro de Desarrollo de Negocios, en este caso, que genera vínculos. Que a parte del profesionalismo que tiene la persona que esta detrás de tu proyecto o que trat a de potenciar algunos sectores de tu empresa, va muy fuerte el tema de los nexos que se generan."

P.A. 34 años, hombre.

En general, ya sea por medio del Centro de Desarrollo de Negocios o por sus propios medios, los empresarios entrevistados ven en la vinculación una oportunidad de gran valor para potenciar a innovación y facilitar esta tarea. Entre otros elementos facilitadores nombrados por un número menor de entrevistados son por ejemplo herramientas como Internet o sistemas de software, o el apoyo de personas del entorno personal, como amigos y familia. Además de dos empresarios $(17 \%)$ que nombran el financiamiento de instituciones públicas como agente facilitador, pero solo uno de ellos ( $8 \%$ ) lo menciona dándole protagonismo antes que otros tipos de apoyo. Al respecto, la frase recogida indica lo siguiente:

\section{"Mis hijos, mi familia. Mi familia somos bien aclanados así que nos yudamos mucho."}

L.T. 58 años, mujer.

\section{Conclusiones}

La innovación no es un tema ajeno o desconocido para los microempresarios entrevistados. Todos manejan ciertos conocimientos sobre el concepto y lo que es muy interesante es que la consideran importante y necesaria, y están dispuestos a innovar pese a los riesgos que esta práctica pueda involucrar a nivel tanto empresarial como personal.

La percepción que tienen respecto a esta, es de una herramienta que les permite diferenciarse de la competencia y ganar un espacio mayor en el mercado. Es para ellos una inversión para el crecimiento de sus empresas. El tipo de innovación que desarrollan la mayoría de estos empresarios es del tipo incremental y mayormente ligada a los procesos y servicios.

En general, los sujetos estudiados han logrado generar ciertos procedimientos e identificar elementos que les son útiles para la innovación en base a experiencias anteriores de ensayo y error. Así mismo, en general, los entrevistados se mueven en el mercado de manera independiente, incluso solitaria, utilizando internet como principal fuente de información, en especial, portales relacionados a sus rubros e información atingente publicada en redes sociales.

Por otra parte, el factor recurso financiero está presente de forma transversal en todas las dimensiones y categorías analizadas, lo que fue expresado en reiteradas ocasiones por los entrevistados de diversas formas. A cuyo respecto manifiestan no contar con información necesaria, ni con el apoyo o la guía suficiente para innovar en sus empresas. Además, considerando los resultados de este estudio con respecto a la falta de apoyo que se traduce, a su vez, en el desconocimiento que plantean los sujetos acerca de donde o a quien acudir para ser orientados acerca de los procesos de innovación que pretenden llevar a cabo en sus empresas, o bien, implementando y desarrollando ideas innovadoras de negocios, se aprecia una apremiante necesidad de intervención y apoyo de gestores tecnológicos.

A lo largo del proceso de análisis de datos se observa que las experiencias que relatan los entrevistados no se alejan de los datos que entregan las Encuestas Nacionales de Innovación en las empresas pequeñas, medianas y grandes con respecto a los temas consultados, por lo que sería interesante que se puedan incluir las microempresas en futuras encuestas. Finalmente, se considera de especial interés abordar el impacto de las políticas públicas de fomento a la innovación en el segmento de los microempresarios exclusivamente con el objetivo de buscar las causas de la discordancia en las respuestas de los mismos sujetos cuando consideran el acceso 
a beneficios de financiamiento estatal y/o asesoría gratuita como un facilitador y al mismo tiempo percibir como un obstáculo importante la falta de estos elementos para poder innovar.

\section{Referencias}

Cancino, C. A., Bonilla, C. A., \& Vergara, M. (2015). The impact of government support programs for the development of businesses in Chile. Management Decision, 1736-1754.

Centros Chile Sercotec. (4 de diciembre de 2018). Nuestros Servicios: Centro de Desarrollo de Negocios. Obtenido de Centros Chile web site: https://centroschile.sercotec.cl/nuestros-servicios

CEPAL Naciones Unidad. (2002). Análisis de la Política de Fomento a las Pequeñas y Medianas Empresas en Chile. Santiago: Red de Reestructuración y Competitividad.

Chetty S. (1996). The case study method for research in small- and médium - sized firms. International small business journal, vol. 5, octubre - diciembre.

Consejo Nacional para la Competitividad. (2010). Agenda de innovación y competitividad 2010-2020. Santiago: Subdere.

Cornejo, M., Carmona, M., Besoaín, C., \& Manosalva, A. (2012). Trayectorias discursos y prácticas en la Investigación Social Cualitativa en Chile. Forum Qualitative Sozialforschung.

Díaz-Bravo, L., Torruco-García, U., Martínez-Hernández, M. \& Varela-Ruiz, M. (2013). La entrevista, recurso flexible y dinámico. Investigación Educación Médica, 162-167. Dini, M., \& Stumpo, G. (2018). MIPYMES en America Latina, un frágil desempeño $y$ nuevos desafíos para las políticas de fomento. Santiago: CEPAL.

Eisenhardt, K. M. (1989). Building Theories from Case Study Research, Academy of Management Review, 14 ( 4): 532-550.

Efrench-Davis, R \& Díaz, Á (abril de 2019). La inversion productiva en el desarrollo económico de Chile: productiva en el desarrollo economico de Chile: evolución y desafíos. Revista de la CEPAL, págs. 28-53.
Flick, U. (2004). Introducción a la investigación cualitativa. 2004). Introduccion a adrid: la investigación nstituto Nacional de Estadísticas. (22 de Abril de 2019). INE: Encuesta Nacional de Innovación. Obtenido de Encuesta Nacional de Innovación: https://www.ine.cl/ estadisticas/economicas/encuesta-nacional-de-innovacion

Glaser, B. \& Strauss, A. (1967). The discovery of grounded theory. Chicago: Aldine Press. transformation (Research Paper 2008/o62). Helsinki: unu-wider.
trant
Krippendorf, K. (1990). Metodología de Análisis de Contenido: Teoría y Práctica. Barcelona: Editorial Paidos. Lewis, W A (1954). Economic development with unlimited supplies of labour. The Manchester School, 28(2), 139-191. Martínez-Salgado, C. (2012) El muestreo en investigación cualitativa.
Principios controversias. Ciência \& Saúde Coletiva , 613-619. Ministerio de Economía Fomento y Turismo. (2017). Informe de resultados: Empresas en Chile. Santiago: División de Politica Comercial e Industria. Ministerio de Economía, Fomento y Turismo. (Febrero de 2018). 10ma Encuesta nacional de innovción en empresas 20152016. Recuperado el Julio de 2018, de www.economia. gob.cl: uploads/2018/o2/Beamer_Innova_2015_2016-3.pdf Ministerio de Economía, Fomento y Turismo. (2019). Boletín ELE -5: Principales resultados. Santiago: Unidad de Estudios, División de Política Comercial e Industrial. Montoya, O. (2004). Schumpeter, Innovación y determinismo tecnológico. Scientia et Technica, 209-213. OCDE. (2005). Manual de OSLO. Guía para la recogida e interpretación de datos sobre innovación. Empresa de Transformación Agraria S.A.

Patton, M. (1990). Qualitative evaluation and research methods. SAGE, 169-186. Paula, L., Alvarez, D., \& Rezende, A. (2018). Innovation and enterpreneurship: successful experiences in Brazil and Peru. European Conference on Innovation and Entrepreneurship; Reading, 564-571. Ranis, G., y Fei, J. C. (1961). A theory of economic development. American Economic Review, 51, 533-565.

Rialp, A. (1998): "El método del caso como técnica de investigación y su aplicación al estudio de la función directiva", ponencia presentada en el IV Taller de Metodología ACEDE, 23-25 de abril, Arnedillo, La Rioja.

Rothman KJ, Greenland S, editores. Modern Epidemiology. $2^{\underline{a}}$ ed. Filadelfia: Lippincott-Raven; 1998 p. 359-432. Schumpeter, J. A. (1942). Capitalism, Socialism and Democracy. London: Taylor \& Francis e-Library. Shaw, E. (1999). A guide to the Qualitative Research Process: Evidence from a Small Firm Study. Qualitative Market Research: An International Journal, 2 (2): 59-70. Sherman, R., \& Webb, R. (1988). Qualitative Research in Education. Londres: Taylor \& Francis, Strauss, A., Corbin, J. (1990). Basics of qualitative research. P edición. Thousand Oaks: Sage.Yin, R.K.(1994). CaseStudy Research-Designand Methods, Applied Social Research Methods (Vol. 5, 2nd ed.), Newbury Park, CA, Sage. 